\title{
Severe acute respiratory syndrome coronavirus 2: An up-to-date review focusing on its treatment options and evolution
}

\author{
Xirui Zhang ${ }^{1}$, Xiao-Hu Wang ${ }^{2}$, Zipeng Yang${ }^{1}$, Tanghui Liu ${ }^{1}$, Zhaowen Ren ${ }^{1}$, Hao Yuan ${ }^{3}$, \\ Xiu-Xiang Zhang ${ }^{4}$, Yasser S.Mahmmod ${ }^{5}$, and Ziguo Yuan ${ }^{1}$ \\ ${ }^{1}$ South China Agricultural University \\ ${ }^{2}$ Guangdong Academy of Agricultural Sciences \\ ${ }^{3}$ Qiqihar Institute of Engineering, Qiqihar, Heilongjiang Province \\ ${ }^{4}$ South China Agricultural University College of Agriculture \\ ${ }^{5}$ Zagazig University
}

April 28, 2020

\begin{abstract}
In December 2019, an unknown pneumonia-disease outbreak emerged in in Wuhan, China. The incriminated causative pathogen, later termed SARS-CoV-2, was found to be a novel lineage of betacoronavirus, belonging to subgenus sarbecovirus. Although China has roughly controlled the epidemic, but the situation in the rest of the world is becoming less and less optimistic. Scientists and pharmaceutical companies around the world are actively looking for treatment options. Till now, there are couple of drugs undergo the clinical trial including convalescent plasma, which possess valid methods of treating or preventing the disease. And since considerable studies had already been carried out based on the analysis of SARS-CoV-2's genome sequence and its comparison with that of the other SARS-like virus, let us review in detail and, hopefully, they might provide us with some key features for answering some of the crucial questions relating to its origin, epidemiology, and treatment to better contain the virus. Basing on that, clues on how to develop a fast and accurate viral diagnostic method and the way of treating it may also be provided. Up to now, the treating option constrained only in a few drugs (Remdesivir, Chloroquine, Hydroxychloroquine, Favipiravir etc.), in addition to the Chinese herb as well as convalescent plasma. More drugs are only roughly screened by virtual screening, and they are still far from not achieving the minimum standards for clinical treatment application. Also, the expected vaccine was reported but it just started the very first trial and still needs time to apply on a large scale. Although the very origin of this virus has been determined to be bats, but till now we still can't determine what kind of animal plays the role of the intermediate host, leaving an issue that needs a further investigation.
\end{abstract}

Severe acute respiratory syndrome coronavirus 2: An up-to-date review focusing on its treatment options and evolution

\section{A review on treatment and evolution of COVID-19}

Xirui Zhang ${ }^{1,2,3,4}$, Xiao-Hu Wang ${ }^{5}$, Zipeng Yang ${ }^{1}$, Tanghui Liu ${ }^{1}$, Zhaowen Ren ${ }^{1}$, Hao Yuan ${ }^{1,6}$, XiuXiang Zhang ${ }^{7}$, Yasser S. Mahmmod ${ }^{8,9}$, Zi-Guo Yuan $^{1,2,3,4}$

${ }^{1}$ College of Veterinary Medicine, South China Agricultural University, Guangzhou, Guangdong Province, PR China

${ }^{2}$ Guangdong Laboratory for Lingnan Modern Agriculture, Guangzhou, Guangdong, PR China

${ }^{3}$ Key Laboratory of Zoonosis Prevention and Control of Guangdong Province, PR China 
${ }^{4}$ Key Laboratory of Zoonosis of Ministry of Agriculture and Rural Affairs, South China Agricultural University, Guangzhou, Guangdong, PR China

${ }^{5}$ Institute of Animal Health, Guangdong Academy ofAgricultural Sciences, Guangzhou, Guangdong Province 510640, PR China

${ }^{6}$ Qiqihar Institute of Engineering, Qiqihar, Heilongjiang Province, PR China

${ }^{7}$ College of Agriculture, South China Agricultural University, Guangzhou, Guangdong Province, PR China

${ }^{8}$ Infectious Diseases, Department of Animal Medicine, Faculty of Veterinary Medicine, Zagazig University, Zagazig, Sharkia, Egypt

${ }^{9}$ Veterinary Sciences Division, Al Ain Men's College, Higher Colleges of Technology, Al Ain, Abu Dhabi, United Arab Emirates

\section{Correspondence:}

Prof Z-G Yuan, College of Veterinary Medicine, South China Agricultural University, Guangzhou, Guangdong Province, PR China; Y-S Mahmmod, Infectious Diseases, Department of Animal Medicine, Faculty of Veterinary Medicine, Zagazig University, Zagazig, Sharkia, Egypt; A/Prof X-X Zhang, College of Agriculture, South China Agricultural University, Guangzhou, Guangdong Province, PR China

Email: ziguoyuan@scau.edu.cn; yasserpcr@gmail.com; xiuxiangzh@scau.edu.cn

\section{Summary}

In December 2019, an unknown pneumonia-disease outbreak emerged in in Wuhan, China. The incriminated causative pathogen, later termed SARS-CoV-2, was found to be a novel lineage of betacoronavirus, belonging to subgenus sarbecovirus. Although China has roughly controlled the epidemic, but the situation in the rest of the world is becoming less and less optimistic. Scientists and pharmaceutical companies around the world are actively looking for treatment options. Till now, there are couple of drugs undergo the clinical trial including convalescent plasma, which possess valid methods of treating or preventing the disease. And since considerable studies had already been carried out based on the analysis of SARS-CoV-2's genome sequence and its comparison with that of the other SARS-like virus, let us review in detail and, hopefully, they might provide us with some key features for answering some of the crucial questions relating to its origin, epidemiology, and treatment to better contain the virus. Basing on that, clues on how to develop a fast and accurate viral diagnostic method and the way of treating it may also be provided.

Up to now, the treating option constrained only in a few drugs (Remdesivir, Chloroquine, Hydroxychloroquine, Favipiravir etc.), in addition to the Chinese herb as well as convalescent plasma. More drugs are only roughly screened by virtual screening, and they are still far from not achieving the minimum standards for clinical treatment application. Also, the expected vaccine was reported but it just started the very first trial and still needs time to apply on a large scale. Although the very origin of this virus has been determined to be bats, but till now we still can't determine what kind of animal plays the role of the intermediate host, leaving an issue that needs a further investigation.

\section{Key words:}

Severe acute respiratory syndrome coronavirus 2; SARS-CoV-2; COVID-19; Betacoronavirus; Treatments; Evolution

\section{Introduction}

Coronaviridae family is a single-strand, positive-sense, enveloped RNA virus family(Su et al., 2016) that can be found circulating in a wide range of avian and mammal species hosts.(Cavanagh, 2007; Ismail, Tang, \& Saif, 2003) In December 2019, a patient was found to have pneumonia caused by an unknown betacoronavirus. By using unbiased sequencing of the sample from the patient, a novel coronavirus was determined. According to the latest official name by the International Committee on Taxonomy of Viruses 
(ICTV), it should be called Severe Acute Respiratory Syndrome Coronavirus 2, SARS-CoV-2 in abbreviation. Then within the same month, in late December, there are already been a crowd of patients that had been diagnosed with infecting the pathogen. (W. M. H. Commission, 2019; Zhu et al., 2020) The widely believed origin, seafood wholesale market in Wuhan, Hubei Province, China, was proven wrong according to the official announcement.(rfi, 2020) The origin of the virus is still a mystery.

SARS-CoV-2 is identified to be a betacoronavirus that belongs to the subgenus sarbecovirus of Coronaviridae family (Beta-CoV lineage B) based on the Phylogenetic analysis.(David S. Hui et al., 2020; R. Lu et al., 2020; Wong, Li, Lau, \& Woo, 2019) Therefore, similar to other coronaviruses, it is a positive-sense single-stranded RNA (+ssRNA) virus with an envelope, and its granules are round or oval in shape with a diameter of 50 to $200 \mathrm{~nm}$.(Zhe, 2020) Typical betacoronavirus structures (S gene, E gene and M gene etc.) was found by Zhu et al.(Zhu et al., 2020) and Zhou et al.(P. Zhou et al., 2020a) Notably, S (spike) protein is one of the main proteins of the virus with its gene coding always used for virus typing.(Zhe, 2020) The protein itself could be divided into two domains, S1 domain and S2 domain. Each domain is responsible for different functions, where $\mathrm{S} 1$ domain is responsible for receptor binding and $\mathrm{S} 2$ domain is responsible for cell membrane fusion.(He et al., 2004; F. Li, 2016) The protein also plays a key role in determining the host's ability of transmission and tropism.(G. Lu, Wang, \& Gao, 2015; Q. Wang, Wong, Lu, Yan, \& Gao, 2016)

It has been confirmed that, through numerous reports from all over the world, the virus has a person-toperson transmission route.(Chan et al., 2020; Rothe et al., 2020; Wired., 2020; Zhe, 2020) The sources of infection seen so far are mainly patients with new coronavirus infection. Asymptomatic infection can also be a source of infection.(N. H. Commission, 2020) Respiratory droplets and close contact transmission are the main routes of transmission. There is the possibility of aerosol transmission in a relatively closed environment for a long-time exposure to high concentrations of aerosol. Population in crowdedness is also under risk is generally susceptible.(N. H. Commission, 2020)

According to the up-to-date studies, the basic reproduction number (R0) of the virus has been estimated to be $1.4-3.9$ with the incubation period is about 1-14 days, mostly 3-7 days, and an average 4.8 days. (Q. Li et al., 2020; Riou \& Althaus, 2020; Rothe et al., 2020) The fatality rate is about $2 \%$ with fever, dry cough and fatigue as the main manifestations. Its viral diagnostic method had already been developed basing on PCR and RT-PCR.(Corman et al., 2020; Jonsdottir \& Dijkman, 2016; Liu et al., 2019; Zhu et al., 2020) Since the E gene and the RdRp gene are comparatively more sensitive, they were chosen for further evaluation for detection.(Corman et al., 2020) Whist, development of its vaccine is also already on the way.(Steenhuysen, 2020)

As of 12 April 2020, the Coronavirus disease 2019 (COVID-19) Situation Report from WHO(WHO, 2020) reported that 1,610,909 confirmed (89,657 new) and 99,690 deaths (6,892 new) cases globally, and 83,369 confirmed (64 new) in China with 3,349 deaths (4 new). These figures show that China has roughly controlled the epidemic, but the situation in the rest of the world is becoming less and less optimistic as there are more concentrated outbreaks are taking place.

Since considerable studies had already been carried out based on the analysis of SARS-CoV-2's genome sequence and its comparison with that of the other SARS-like virus, let us review in detail and, hopefully, they might provide us with some key features for answering some of the crucial questions relating to its origin, epidemiology, and treatment to better contain the virus. Basing on that, clues on how to develop a fast and accurate viral diagnostic method and the way of treating it may also be provided.

\section{Clinical manifestations \& Pathological features}

Based on the current epidemiological investigation fever, dry cough and fatigue are the main manifestations. A few patients have symptoms such as nasal congestion, runny nose, sore throat, myalgia and diarrhoea.(N. H. Commission, 2020) Severe patients usually have dyspnea and/or hypoxemia one week after the onset of symptoms, and severe patients can quickly progress to acute respiratory distress syndrome, septic shock, difficult to correct metabolic acidosis, and coagulation dysfunction and functional failure of multiple organs.(N. H. Commission, 2020) It is worth noting that the course of severe and critically ill patients can display a 
moderate or low fever, and even no obvious fever. Mild patients show only low fever and mild fatigue with no pneumonia manifestations. From the current cases, most patients have a good prognosis with only a few patients have a critical condition. The prognosis for the elderly and those with chronic underlying disease is poor. Symptoms in children are relatively mild.(N. H. Commission, 2020)

The pathological features of COVID-19 are very similar to those in SARS and MERS coronavirus infections.(Z. Xu et al.) Besides, liver biopsy specimens from patients with COVID-19 showed moderate microvascular steatoplasty and mild active inflammation of the hepatic lobular manifold area, suggesting that the injury may be due to SARS-CoV-2 infection or drug-induced liver injury. There was a small amount of inflammatory infiltration of mononuclear cells in the myocardial interstitium, but no other myocardial parenchymal damage was found.(Z. Xu et al.) The number of CD4+ and CD8+ cells in peripheral blood was greatly reduced, but their states were over-activated. In addition, CCR $4+$ CCR $6+$ Th17 cells with a high pro-inflammatory effect were increased. CD8 $+\mathrm{T}$ cells were found to have high concentrations of cytotoxic granules.(Z. Xu et al.)

To be short, in a study by $\mathrm{Xu}$ et al.(Z. Xu et al.), the chest radiograph showed a rapid progression of pneumonia with slight differences between the two lungs. Also, liver tissue exhibits moderate microvascular steatoplasty and mild lobular active inflammation, but there is no firm evidence to support SARS-CoV-2 virus infection or drug-induced liver damage. There were no obvious histological changes in the heart tissue, suggesting that SARS-CoV-2 infection may not directly damage the heart. Lymphopenia is a common feature in patients with COVID-19.

Moreover, another study had also provided some insight of the pathological findings of the SARS-CoV-2. A study by Mao et al.(Mao et al., 2020) found that severe patients often have neurological symptoms, compared with non-severe COVID-19 patients, with acute cerebrovascular disease, disturbance of consciousness, and skeletal muscle symptoms. After analysing 522 samples from the SARS-CoV-2 patients, Diao et al.(Diao et al., 2020) discovered that SARS-CoV-2 patients, especially elderly patients (over 60 years of age) and patients in need of intensive care unit (ICU) care, had a significant reduction in the total number of T cells, CD4+, and CD8+ T cells. The number of T cells was negatively correlated with serum IL-6, IL-10 and TNF- $\alpha$ concentrations. The levels of IL-6, IL-10 and TNF- $\alpha$ in patients with the disease declined with the count of $\mathrm{T}$ cell recovered. It is worth mentioning that patients with SARS-CoV-2 had significantly higher levels of PD-1, a marker of T cell depletion. In addition, the expression of PD-1 and Tim-3 from T-cells increased during the shift from the precursor stage to the apparent symptomatic stage, further implied a decrease in T-cells and functional depletion in SARS-CoV-2 patients. Finally, a study by Cheng et al.(Cheng et al., 2020) had discovered patients with kidney injury have a higher risk of in-hospital death after analysing 710 cases of SARS-CoV-2 patients.

\section{Where did it come from?}

Although the natural reservoir of the virus is believed to be bats with a $96 \cdot 2 \%$ sequence identity, as it's reported by Shi et al.(P. Zhou et al., 2020b), however, its intermediate host is not yet determined.

Experiment carried out by researchers from HK (Lam et al.) also displayed $85.5 \%$ to $92 \cdot 4 \%$ sequence identity with that of the pangolin-derived coronavirus, together with some other evidence suggesting that Malayan pangolins in southern China could be the intermediate host.(Lam et al., 2020)

However, plenty of issues are still vague, whereas the theory itself still is not fully proved. Some scholar points out that the result only indicates that the virus is possibly originated from pangolins but not firmly confirm the pangolin must be the intermediate host. Lam's research group also stated their confusion that the pangolin has actually a comparatively limited population and they are entitled to be endangered in many places and the high sequence identity between the viruses may be explained by convergent evolution. And therefore, further investigation on this subject is needed.

Other than pangolins, snakes and minks was suspected to be the intermediate host for a short period of time, but after an intense debate, all proposal was rejected. 
To be short, most debate about the intermediate host of SARS-CoV-2 can be found on website Biorxiv (https://www.biorxiv.org/). Discussion of which animal is the intermediate host end up with nothing, leaving the question still in mystery. The only thing that had been determined is the virus originates from bats.(P. Zhou et al., 2020b)

\section{Treatments available or under development}

Since the outbreak had dealt substantial damage to the society in the whole world, lots of scientists had started to focus on seeking a viable solution to deal with the disease. Up to now, a considerable number of drugs had already under development and even started the clinical trial.

\section{Drugs reported to be effective}

Since SARS-CoV-2 belongs to the betacoronavirus, therefore, it is reasonable to use drugs used to treat SARS and MERS to inhibit the infection of the novel virus as four key enzymes in the life cycle as well as the Spike protein of the viruses are similar. Those proteins are considered promising targets for drugs.(Clercq, 2020) Whist, some other broad-spectrum antiviral drugs were also reported to be effective (Table 1).

Remdesivir, is an adenosine analogue, an antiviral drug that against a wide range of RNA viruses (includes SARS/MERS(Sheahan et al., 2017)) in both culture cells and animal models, is initially an experimental antiviral drug that treats Ebola, is now under the cooperation of Gilead Sciences Inc. with researchers and clinicians in China. It can cause the RNA chains of the viral to terminate before it gets to its mature stage and therefore fulfills its antiviral function.(Warren et al., 2016) A study by Wang et al.(M. Wang et al., 2020) had shown that Remdesivir can effectively suppress the infection of the virus in the highly sensitive human cell line (human liver cancer Huh-7 cells). From their detection, Remdesivir functions one stage after the virus entry.(M. Wang et al., 2020) The $\mathrm{EC}_{90}$ value of Remdesivir in against the SARS-CoV-2's infection of $\mathrm{E} 6$ cells was $1.76 \mu \mathrm{M}$. Whist, the $\mathrm{EC}_{50}$ value is $0.77 \mu \mathrm{M}(\mathrm{SI}>129.87)$.(M. Wang et al., 2020) Currently, phase III clinical trials of Remdesivir have been launched in China. In America, the first case of SARSCoV-2 patient had been treated with Remdesivir, and now had already been cured.(Holshue et al., 2020) Also, in France, a patient was also treated with Remdesivir and also cured.(Dealmoon, 2020) But further trials are still needed as there are only a few experiments on monkey model in treating MERS but not the SARS-CoV-2.(Dealmoon, 2020)

Chloroquine, is a cheap drug with a long history that until recently been reported to have a broad-spectrum antiviral capability as well as immune-modulating activity(Savarino, Di Trani, Donatelli, Cauda, \& Cassone, 2006; M. Wang et al., 2020; Yan et al., 2013). It is originally used to treat malarial and autoimmune disease and it is currently under evaluation in an open trial (ChiCTR2000029609). Chloroquine inhibits virus's infection of the cells by escalating the endosomal $\mathrm{PH}$ that is essential for virus/cell fusion, and in the meanwhile, it interferes with the glycosylation of the SARS-viruses' receptors.(Vincent et al., 2005) The result of the study by Wang et al. displayed that the drug functions at both the entry and post-entry stages of the virus invasion into the cells.(M. Wang et al., 2020) Moreover, it was reported to be widely spread in the whole body, including the lung.(M. Wang et al., 2020) The $\mathrm{EC}_{90}$ value of Chloroquine in against the SARS-CoV-2's infection of E6 cells was $6.90 \mu \mathrm{M}$, whereas the $\mathrm{EC}_{50}$ value is $1 \cdot 13 \mu \mathrm{M}(\mathrm{SI}>88.50)$. (M. Wang et al., 2020) So far, more than 100 patients had been used Chloroquine Phosphate to treat the disease, there have been no drug-related, obvious adverse reactions. Since it had been used for more than 70 years in treating other diseases, experts are considered it controllable and safe to enlarge the treatment to a wider population.(YSA, 2020)

Hydroxychloroquine, is a 4-aminoquinoline derivative antimalarial drug. Its action and mechanism are similar to Chloroquine, but its toxicity is only half that of Chloroquine. Medical doctors from Wuhan University People's Hospital found that all the patients in their hospital with systemic lupus erythematosus weren't infected with the SARS-CoV-2. Hence, they suspect that this effect may be argued by the long-termed administration of the Hydroxychloroquine to treat systemic lupus erythematosus, which helps in prevention of the infection. It seems reasonable and plausible explanation since Hydroxychloroquine has similar treating effect as Chloroquine does, and Chloroquine, as mentioned above, was reported to be effective in treatment 
of SARS-CoV-2. Therefore, they had treated 20 cases of the patients by using Hydroxychloroquine and found that the clinical symptoms were improved significantly in one to two days. Review of chest CT after five days of the drug use showed a significant improvement in the absorption in 19 cases. The remaining patient (previously had renal insufficiency) had progressive lesions in the chest $\mathrm{CT}$, but the patient's clinical symptoms improved significantly on the second day of Hydroxychloroquine use. In addition, none of the normal patients enrolled in the group developed severe illness, and one of them was discharged/ released on February 13.(Publish, 2020) This result had preliminary confirmed a short-term efficacy of Hydroxychloroquine in the treatment of new coronary pneumonia, which can effectively relieve symptoms, reverse the rate of exacerbation, and shorten the course of the disease. Further application trials of Hydroxychloroquine in severe and critical neo-coronary pneumonia are ongoing.

Favipiravir, is a guanine analog approved for the treatment of influenza, which effectively inhibits the RNA-dependent RNA polymerase of RNA viruses. A recent study reported its activity on SARS-CoV2.(Clercq, 2020; M. Wang et al., 2020) A randomized trial of Favravavir in combination with interferon- $\alpha$ (CHICTR090029) and barbiturvir (CHICTR09009554) for SARS-CoV-2 has been conducted.(Clercq, 2020)

Besides, Chinese traditional medicine (TCM), also known as Chinese herb, was also reported to play a key role in treating the disease. (Hongzhuan, 2020) According to statistics, as of 00:00 on February 5th, the four pilot provinces used TCM to treat 214 confirmed cases had shown an over $90 \%$ rate of effective, of which the symptoms of $60 \%$ of patients experienced significant improvement, $30 \%$ of patients with stable symptoms without exacerbation.(Ping;, 2020)

\section{Candidate drugs not yet proven effective in treatment of SARS-CoV-2}

As some drugs, as mentioned above, were reported to be effective in treating the epidemic, more drugs were merely screened by computer calculating (Table 2). Simply speaking, about 30 drugs was screened out with these crudely selected drugs have chosen different mechanisms and targets (3CL hydrolase/ papain-like protease/ ACE2/ S protein of SARS-CoV-2 etc.) to have the inhibitory effect on the virus, providing a lot of new ideas to fight the novel coronavirus.

\section{Vaccines}

As the drugs are under development, vaccine development had also made a certain progress. Experts from USA and China had cooperated to develop a vaccine against the new virus. Some articles point out that vaccine development against SARS-CoV may face many challenges due to mutation of protein-encoding genes.(Y. Zhou, Yang, Huang, Jiang, \& Du, 2019)

The $\mathrm{S}$ protein is the main target antigen, and the development of recombinant vector vaccines, DNA vaccines and subunit vaccines has been carried out on this basis. Phase I trial was and will be initiated in China(Mak, 2020; NIH, 2020) and America(Dunn, 2020) respectively on testing the newly developed recombinant vaccine (China) and RNA vaccine (America) according to up to date reports.

\section{Monoclonal antibody (mAbs)}

According to a clinical trial carried out by a team from University of Science and Technology of China, a mAbs termed Tocilizumab had proven efficient in treating the SARS-CoV-2's patients.(Hefei, 2020) As reported, Tocilizumab together with conventional treatment, by blocking the inflammatory storm, could prevent patients from transitioning to severe and critical illness, thereby reducing the mortality rate. In phase I of the clinical trial with 14 participant patients, after treated with the new treatment regimen of "Tocilizumab + conventional treatment", the body temperature of all 11 patients with fever symptom returned to normal (14 participant patients in total, 11 with fever); the respiratory oxygenation index improved in vary degrees; 4 patients had displayed a better absorption of CT lesions within their lungs.(Hefei, 2020)

A team from the Fudan University had successfully obtained the first case of human-originated antibody of the novel virus. The team isolated single B cells from samples of convalescent patients of the novel coron- 
avirus, cloned the variable region of the antibody by PCR, and quickly obtained and screened hundreds of human antibodies strains. They found that many of them can recognize the RBD antigen of SARS-CoV-2. (BioArt, 2020) This research provides candidate antibodies for the development of human Neutralization Antibody Test. Also, a company called Vir Biotechnology is currently working on anti-coronavirus monoclonal antibodies (mAbs) that bind and neutralize the SARS-CoV-2.(Biotechnology, 2020)

\section{Convalescent plasma}

According to Dr. Mike Ryan, head of WHO's health emergencies program, convalescent plasma has been proven an "effective and life-saving" solution against other infectious diseases including rabies and diphtheria. "It is a very valid way to explore therapeutics, especially when we don't have vaccines and we don't have specific antivirals." (REUTERS, 2020) In China, clinical studies have shown that convalescent plasma is safe and effective, and it is an effective means for treating severe and critical illness. One of the patients currently being treated has been discharged, and one has been able to walk on the ground. The remaining patients are all in the recovery period. (YSA, 2020)

\section{Evolutionary findings relating to the Novel Coronavirus}

To find out more about its relationship with SARS and MERS, Lu's research group have reported that SARS-CoV-2 have about $79 \%$ sequence identity with SARS-CoV but lower identity with that of the one from MERS-CoV (with only 50\%).(R. Lu et al., 2020; Wu et al., 2020) Classification by Zhou et al. using seven conserved replicase domains in ORF1ab had also indicate that SARS-CoV-2 and SARS-CoV belong to the same species with a $94.6 \%$ amino acid identity.(P. Zhou et al., 2020a)

In the meantime, Study by Zhu et al. had indicated that it has formed an isolated clade, composing with other two virus strains that originated from bats, ZC45 and ZXC21.(Zhu et al., 2020) The result was further proven by the study from Lu et al, which showed thatSARS-CoV-2 have formed a considerable long branch length together with bat-SL-CoVZC45 and bat-SL-CoVZXC21 in a phylogenetic tree that is distinct from SARS.(R. Lu et al., 2020) In Lu's study, samples from the Wuhan patients were examined, the result of an $88 \%$ sequence identity with both bat-SL-CoVZC45 and bat-SL-CoVZXC21 was shown.(R. Lu et al., 2020) Also, a study by Chan et al had demonstrated the same findings by using the samples from patients from Hongkong to do the sequence comparison.(Chan et al., 2020) From the same study examining the HK patients, it was reported that the virus genome is about 29.8 kilobases with $38 \%$ of the genome consist of GC.(Chan et al., 2020) Notably, other two research groups had also reported respectively that the virus genome had about $89 \%$ (Chan et al., 2020) and $86.9 \%$ (Zhu et al., 2020) nucleotide sequence identity to that of the bat SARS-like CoV (bat-SL-CoVZC45) genome. But from a report by Zhou et al, they claimed that a bat-originated coronavirus termed RaTG13 is the closest relative of the virus from Wuhan as its indicated from their study analyzing the RdRp gene and S gene sequence.(P. Zhou et al., 2020a)

Results from Lu's further analysis of the phylogenetic tree has displayed that three clades are formed: clade 1 is composed by SARS-related strains from Rhinolophussp from Bulgaria (accession number GU190215) and Kenya (KY352407); clade 2 is formed by 10 samples from Wuhan and bat-derived strain: bat-SL-CoVZC45 and bat-SL-CoVZXC21, just as mentioned above; and clade 3 is formed by the human-derived SARS-CoV and several bat-derived coronaviruses.(R. Lu et al., 2020) Phylogenetic tree analysis employing Maximum likelihood (ML) methods by Benvenuto et al had shown the same outcome with SARS virus, SARS-like batderived virus and the Novel CoV together formed one clade (termed clade II) and MERS virus themselves forming another (termed clade I).(Benvenuto et al., 2020) Within clade II, Bat-derived SARS-like coronavirus and the SARS-CoV-2 formed IIa cluster and Bat-derived SARS-like coronavirus and the SARS virus formed IIb cluster.

As it is mentioned, $88 \%$ similarity was displayed when comparing the SARS-CoV-2 with bat-derived CoV ZC45 and ZXC21. To dive deeper, five regions of the gene -E, M, 7, N and 14, as its reported, are both have over $90 \%$ sequence identity with $\mathrm{E}$ gene the highest of all (98.7\%).(R. Lu et al., 2020) Meanwhile, prediction of 12 of its coding regions had demonstrated that the genomic organization, as well as the length of those proteins, are alike with only minor variations in comparison to the counterparts from bat-SL-CoVZC45 and 
bat-SL-CoVZXC21.(R. Lu et al., 2020)

Contrastingly, the S gene and protein 13 holds the lowest identity of all, with only about $75 \%$ and $73 \%$ sequence identity no matter comparing with ZC45 or ZXC21.(R. Lu et al., 2020) But from the study by Chan et al, SARS-CoV-2's Spike protein was reported to have a higher nucleotide identity, at about $84 \%$ and $78 \%$ identity with that of the one from bat-SL-CoVZC45 and human SARS coronavirus respectively. (Chan et al., 2020) Zhou et al found SARS-CoV-2's S gene and RdRp gene indeed has a high variation with other $\mathrm{CoVs}$ (less than $75 \%$ in identity), but has a high sequence identity of that two genes with RaTG13 at a figure of $93.1 \%$, and the S gene of RaTG13 and SARS-CoV-2 is much longer than other SARSr-CoVs.(P. Zhou et al., 2020a) But it is worth mentioning that since the spike protein of SARS-like coronavirus is the most variable in the viral genome, the highly differentiated outcome is actually not surprising at all.

As mentioned in the overview, Spike protein has two domains with both of which responsible for some vital functions. Detailed analysis of SARS-CoV-2's difference compares to two bat-derived virus strains batSL-CoVZC45 and bat-SL-CoVZXC21 has shown that it's S1 domain has a comparatively greater different with only $68 \%$ sequence identity, whereas the S2 domain is far more alike with a $93 \%$ identity.(R. Lu et al., 2020) As for the amino acid, about 50 amino acid remain not changed in S1 when comparing with SARS. But from some position of the C-terminal suggest that mutation and deletion happened in the batderived strain.(R. Lu et al., 2020) Despite the whole-genome of SARS-CoV-2 is reported to be closer to the bat-SL-CoVZC45 and bat-SL-CoVZXC21, its receptor binding domain is closer to that of the one from SARS-CoV. This result is further proven by a following three-dimensional modelling verification. Similar to other betacoronaviruses, the receptor binding domain still composed by a core domain and an external subdomain but the external subdomain is more similar to that of the SARS-CoV.(R. Lu et al., 2020) Since the receptor domain's similarity is close to the SARS-CoV, it gives the hint that ACE2 might still be the possible mechanism for the cell entry. Through the protein modelling experiment and structural analysis of the receptor binding domain $(\mathrm{RBD})$, it shows that it still preserves sufficient affinity to the Angiotensin converting enzyme 2 (ACE2) receptor and still be able to use it as a mechanism of cell entry. Thus, it may enhance the human-to-human transmission(Ge et al., 2013; Hu et al., 2017; X. L. Yang et al., 2015), despite some difference of the composing amino acids that was identified after comparing of the proteins from SARS through homology modelling. Interestingly, the result from the study by Chan et al.(Chan et al., 2020), had reported that the amino acid sequence identity of the N-terminal of the subunit 1 is around $66 \%$, the core domain is about $68 \%$. But the protein sequence has only about $39 \%$ in identity with SARS-CoV. Depending on this outcome, they assumed some change of the entry mechanism might take place instead of the traditional mechanism.(Chan et al., 2020)

Conserved replicase domains (ORF 1ab) of the novel coronavirus was reported to be less than $90 \%$ in the identity with other betacoronavirus with 1 b (about 86\%) slightly lower than that of 1a (about 90\%). (Zhu et al., 2020) A supplementary analysis of the main encoding regions of the typical members of the subgenus sarbecovirus indicates that recombination of the virus's gene might have happened in gene $1 \mathrm{~b}$ as the novel virus cluster with two other closest bat-derived virus strains (bat-SL-CoVZC45, and bat-SL-CoVZXC21) in the tree of $1 \mathrm{a}$ and $\mathrm{S}$ gene, as suggested in the phylogenetic analysis, but not clustering in the tree of $1 \mathrm{~b}$. Topological position change had also been detected as it is announced by Wu et al.(Wu et al., 2020), and therefore, it suggests the same conclusion of recombination within the subgenus Sarbecovirus from bats.

The findings of Lu's study revealed that SARS-CoV-2 was distinct from SARS-CoV in a phylogeny of the complete RNA-dependent RNA polymerase (RdRp) gene.(R. Lu et al., 2020) Nevertheless, SARS-CoV-2's RdRp region had shown a high sequence similarity (96.2\% whole-genome identity) with the bat-originated coronavirus BatCoV RaTG13 according to the study by Zhou et al.(P. Zhou et al., 2020a) By using a couple of aligned sequences, they discovered that there was no recombination took place in the genome of SARSCoV-2(P. Zhou et al., 2020a), which further escalated the result by the team of Lu et al which assumed that it is the bat-derived virus that had the recombination rather than the one from Wuhan patient.

\section{Concluding remarks}


SARS-CoV-2 that causes an epidemical pneumonia outbreak in Wuhan is evident to be a novel betacoronavirus that belongs to the subgenus Sarbecovirus through various analysis(D. S. Hui et al., 2020; R. Lu et al., 2020; Wong et al., 2019). Controlling its spread is becoming a more and more urgent affair to deal with for the whole world. Though its mortality rate is only about $2 \%$, but still few treatment options is available. Up to now, the treating option constrained only in a few drugs (Remdesivir, Chloroquine, Hydroxychloroquine, Favipiravir etc.), in addition to the Chinese herb as well as convalescent plasma. More drugs are only roughly screened by virtual screening, and they are still far from not achieving the minimum standards for clinical treatment application. Also, the expected vaccine was reported but it just started the very first trial and still needs time to apply on a large scale. Before the full development of an ideal preventive and/or treatment, it would be a tough time for the human race.

It is worth noting that older men with SARS-CoV-2 infection and other underlying diseases typically have a higher mortality rate than older women or younger, much healthier patients; (Chen et al.; Huang et al.; F.-S. Wang \& Zhang, 2020) Further studies are still needed to determine the relevant influencing factors for this finding.

As the SARS-CoV-2 belongs to Nidovirales together with other two serious respiratory diseases, a comparison of its nucleotide sequence and construction of a phylogenetic tree with SARS and MERS has been done. The findings of these analyses indicated that the SARS-CoV-2 is comparatively closer to SARS but still distinct from it.(R. Lu et al., 2020; Wu et al., 2020; P. Zhou et al., 2020a) Evidence was provided that the virus has molecular and structural changes as well as some sites under positive pressure, and moreover, its Spike Glycoprotein and nucleocapsid protein has mutated.(Benvenuto et al., 2020)

It's widely believed that it is close to two bat-derived virus strains; bat-SL-CoVZC45 and bat-SL-CoVZXC21.(Chan et al., 2020; R. Lu et al., 2020; Zhu et al., 2020) But a finding from Zhou et al suggests the most similar one is the bat-derived strain called RaTG13.(P. Zhou et al., 2020a) Although the very origin of this virus has been determined to be bats, but till now we still can't determine what kind of animal plays the role of the intermediate host, some say pangolin, some say others, leaving an issue that needs a further investigation.

Deletion and mutation were detected in the C-terminal of S region of the gene, meanwhile, some insertion and replacement were also detected in its N-terminal. Three-dimensional modelling, structural analysis and amino acid sequencing had together verified the receptor binding domain is still capable of binding with Angiotensin converting enzyme 2 (ACE2), indicating the cell entry mechanism relying on ACE2 still possible.(Chan et al., 2020; R. Lu et al., 2020; P. Zhou et al., 2020a)

When studying the ORF1ab and RdRp gene, it was found that recombination took place in $1 \mathrm{~b}$ in bat-derived strains as it is indicated from the phylogenetical analysis together with topological findings in ORF1ab.(R. Lu et al., 2020; Wu et al., 2020) And by analysing the alignment of the RdRp gene, it further confirmed that there is indeed some recombination happened in the $1 \mathrm{~b}$ of bats.(P. Zhou et al., 2020a)

\section{Ethical Statement}

The authors confirm that the ethical policies of the journal, as noted on the journal's author guidelines page, have been adhered to. No ethical approval was required as this is a review article with no original research data.'

\section{Data Availability Statement}

Data sharing is not applicable to this article as no new data were created or analyzed in this study.

\section{Acknowledgments}

Thank you for the support from the public experimental instrument platform of Veterinary College of South China Agricultural University.

\section{Contributors}


Conceptualization, Z-GY and X-XZ; writing - original draft preparation, XZ, X-HW, ZY and HY; writingreview and editing, Y-SM, X-XZ, and Z-GY; writing- information collection and tabulation, ZR, and TL. All authors approved and signed off the final version.

\section{Fundings}

This research was funded by Novel Coronavirus Pneumonia Emergency Key Technologies R \& D Program of Foshan; the National Natural Science Foundation of China, grant number 31972707, the Natural Science Foundation of Guangdong Province, grant number 2019A1515011534.

\section{Conflicts of Interest}

The authors declare no conflict of interest.

\section{References}

Benvenuto, D., Giovannetti, M., Ciccozzi, A., Spoto, S., Angeletti, S., \& Ciccozzi, M. (2020). The 2019new coronavirus epidemic: evidence for virus evolution. Journal of Medical Virology, 10.1002/jmv.25688. doi:10.1002/jmv.25688

BioArt. (2020). Fudan University and Ai Yue Biological get the first case of Novel coronavirus humanoriginated antibody.

Biotechnology, V. (2020). Vir Biotechnology applying multiple platforms to address public health risk from Wuhan coronavirus.

Cavanagh, D. (2007). Coronavirus avian infectious bronchitis virus. Vet Res, 38 (2), 281-297. doi:10.1051/vetres:2006055

Chan, J. F.-W., Yuan, S., Kok, K.-H., To, K. K.-W., Chu, H., Yang, J., . . Yuen, K.-Y. (2020). A familial cluster of pneumonia associated with the 2019 novel coronavirus indicating person-to-person transmission: a study of a family cluster. Lancet (London, England), S0140-6736(0120)30154-30159. doi:10.1016/S01406736(20)30154-9

Chen, N., Zhou, M., Dong, X., Qu, J., Gong, F., Han, Y., . . . Zhang, L. Epidemiological and clinical characteristics of 99 cases of 2019 novel coronavirus pneumonia in Wuhan, China: a descriptive study. The Lancet . doi:10.1016/S0140-6736(20)30211-7

Cheng, Y., Luo, R., Wang, K., Zhang, M., Wang, Z., Dong, L., . . Xu, G. (2020). Kidney impairment is associated with in-hospital death of COVID-19 patients. medRxiv , 2020.2002.2018.20023242. doi:10.1101/2020.02.18.20023242

Clercq, G. L. E. D. (2020). Therapeutic options for the 2019 novel coronavirus (2019-nCoV). Retrieved fromhttps://www.nature.com/articles/d41573-020-00016-0

Commission, N. H. (2020). New Coronavirus Pneumonia Treatment. Retrieved fromhttp://117.136.191.144/cache/www.nhc.gc args2=242-27110806003998_d8735446fe3f262c3af65afccabb7c46_10001002_9c896c2ed0c4f3d8943f518939a83798_78e20dbf6e0e320328c6c4048386abde

Commission, W. M. H. (2019). Report of clustering pneumonia of unknown etiology in Wuhan City.

Corman, V. M., Landt, O., Kaiser, M., Molenkamp, R., Meijer, A., Chu, D. K., . . . Drosten, C. (2020). Detection of 2019 novel coronavirus (2019-nCoV) by real-time RT-PCR. Euro surveillance : bulletin Europeen sur les maladies transmissibles = European communicable disease bulletin, 25 (3), 10.2807/15607917.ES.2020.2825.2803.2000045. doi:10.2807/1560-7917.ES.2020.25.3.2000045

Dealmoon. (2020). Good news from the American anti-pneumonia magic drug! On the same day, terror cruise ships surged by 70 new cases overnight.

Diao, B., Wang, C., Tan, Y., Chen, X., Liu, Y., Ning, L., . . Chen, Y. (2020). Reduction and Functional Exhaustion of T Cells in Patients with Coronavirus Disease 2019 (COVID-19). medRxiv , 2020.2002.2018.20024364. doi:10.1101/2020.02.18.20024364 
Dunn, A. (2020). The first study of a potential coronavirus vaccine will soon start recruiting healthy volunteers in Georgia at Emory University. Retrieved fromhttps://www.businessinsider.com/coronavirus-vaccinetesting-first-clinical-trial-expands-to-georgia-2020-3

Fan, Y. (2020). Shanghai Institute of Materia Medica: 30 drugs may have therapeutic effects on new coronavirus. Retrieved fromhttps://www.thepaper.cn/newsDetail_forward_5633662

Ge, X. Y., Li, J. L., Yang, X. L., Chmura, A. A., Zhu, G., Epstein, J. H., . . Shi, Z. L. (2013). Isolation and characterization of a bat SARS-like coronavirus that uses the ACE2 receptor. Nature, 503 (7477), 535-538. doi:10.1038/nature12711

He, Y., Zhou, Y., Liu, S., Kou, Z., Li, W., Farzan, M., \& Jiang, S. (2004). Receptor-binding domain of SARS-CoV spike protein induces highly potent neutralizing antibodies: implication for developing subunit vaccine. Biochem Biophys Res Commun, 324 (2), 773-781. doi:10.1016/j.bbrc.2004.09.106

Hefei, P. s. G. o. (2020). Important progress has been made in the research of new crown pneumonia at the University of Science and Technology of China!

Holshue, M. L., DeBolt, C., Lindquist, S., Lofy, K. H., Wiesman, J., Bruce, H., . . Pillai, S. K. (2020). First Case of 2019 Novel Coronavirus in the United States. New England Journal of Medicine . doi:10.1056/NEJMoa2001191

Hongzhuan, C. L. G. G. R. Y. F. W. Z. M. Z. Y. f. Y. W. L. X. Z. L. Z. H. C. (2020). Application and research progress of traditional Chinese medicine in prevention and treatment of coronavirus disease 2019.Academic Journal of Shanghai University of Traditional Chinese Medicine, 1-8.

Hu, B., Zeng, L. P., Yang, X. L., Ge, X. Y., Zhang, W., Li, B., . . Shi, Z. L. (2017). Discovery of a rich gene pool of bat SARS-related coronaviruses provides new insights into the origin of SARS coronavirus.PLoS Pathog, 13 (11), e1006698. doi:10.1371/journal.ppat.1006698

Huang, C., Wang, Y., Li, X., Ren, L., Zhao, J., Hu, Y., . . . Cao, B. Clinical features of patients infected with 2019 novel coronavirus in Wuhan, China. The Lancet . doi:10.1016/S0140-6736(20)30183-5

Hui, D. S., E, I. A., Madani, T. A., Ntoumi, F., Kock, R., Dar, O., . . Petersen, E. (2020). The continuing 2019-nCoV epidemic threat of novel coronaviruses to global health - The latest 2019 novel coronavirus outbreak in Wuhan, China. Int J Infect Dis, 91 , 264-266. doi:10.1016/j.ijid.2020.01.009

Hui, D. S., I Azhar, E., Madani, T. A., Ntoumi, F., Kock, R., Dar, O., . . Petersen, E. (2020). The continuing 2019-nCoV epidemic threat of novel coronaviruses to global health - The latest 2019 novel coronavirus outbreak in Wuhan, China. International journal of infectious diseases : IJID : official publication of the International Society for Infectious Diseases, 91 , 264-266. doi:10.1016/j.ijid.2020.01.009

Ismail, M. M., Tang, A. Y., \& Saif, Y. M. (2003). Pathogenicity of turkey coronavirus in turkeys and chickens. Avian Dis, 47 (3), 515-522. doi:10.1637/5917

Jonsdottir, H. R., \& Dijkman, R. (2016). Coronaviruses and the human airway: a universal system for virus-host interaction studies. Virol J, 13 , 24. doi:10.1186/s12985-016-0479-5

Lam, T. T.-Y., Shum, M. H.-H., Zhu, H.-C., Tong, Y.-G., Ni, X.-B., Liao, Y.-S., . . Guan, Y. (2020). Identifying SARS-CoV-2 related coronaviruses in Malayan pangolins. Nature . doi:10.1038/s41586-020-21690

Li, F. (2016). Structure, Function, and Evolution of Coronavirus Spike Proteins. Annu Rev Virol, 3 (1), 237-261. doi:10.1146/annurev-virology-110615-042301

Li, Q., Guan, X., Wu, P., Wang, X., Zhou, L., Tong, Y., . . Feng, Z. (2020). Early Transmission Dynamics in Wuhan, China, of Novel Coronavirus-Infected Pneumonia. N Engl J Med . doi:10.1056/NEJMoa2001316

Liu, G. S., Li, H., Zhao, S. C., Lu, R. J., Niu, P. H., \& Tan, W. J. (2019). Viral and Bacterial Etiology of Acute Febrile Respiratory Syndrome among Patients in Qinghai, China. Biomed Environ Sci, 32 (6), 
438-445. doi:10.3967/bes2019.058

Lu, G., Wang, Q., \& Gao, G. F. (2015). Bat-to-human: spike features determining 'host jump' of coronaviruses SARS-CoV, MERS-CoV, and beyond.Trends Microbiol, 23 (8), 468-478. doi:10.1016/j.tim.2015.06.003

Lu, R., Zhao, X., Li, J., Niu, P., Yang, B., Wu, H., . . . Tan, W. (2020). Genomic characterisation and epidemiology of 2019 novel coronavirus: implications for virus origins and receptor binding.Lancet (London, England), S0140-6736(0120)30251-30258. doi:10.1016/S0140-6736(20)30251-8

Mak, E. (2020). China approves first homegrown COVID-19 vaccine to enter clinical trials. Retrieved fromhttps://www.bioworld.com/articles/433791-china-approves-first-homegrown-covid-19-vaccine-to-enter-clinicaltrials

Mao, L., Wang, M., Chen, S., He, Q., Chang, J., Hong, C., . . Hu, B. (2020). Neurological Manifestations of Hospitalized Patients with COVID-19 in Wuhan, China: a retrospective case series study.medRxiv , 2020.2002.2022.20026500. doi:10.1101/2020.02.22.20026500

NIH. (2020). A Phase I Clinical Trial in 18-60 Adults (APICTH).

Ping;, Y. M. S. M. W. T. G. L. W. Z. H. (2020). Discussion on Traditional Chinese Medicine Treatment of New Coronavirus Pneumonia from the Perspective of "Three Causes and Measures". Chin J Geriatrics Res (Electronic Edition), 1-001. doi:10.3877/cma.j.issn.2095-8757.2020.01.E001

Publish, W. (2020). Lupus erythematosus treatment has significant short-term efficacy in treating new coronary pneumonia. Retrieved fromhttps://www.toutiao.com/i6794705852260418052/?tt_from=copy_link\&iutm_-

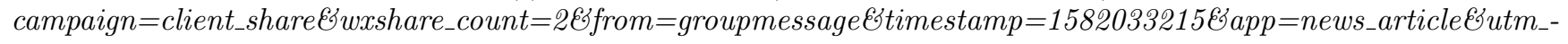
source $=$ copy_linkEisappinstalled=0Ëutm_medium =toutiao_iosEGreq_id=202002182140140101310741551B04F15FËgroup_$i d=67947058522604180528$ pbid $=6662803681090733575$

qi, W. (2020). Major achievement of Academician Li Lanjuan's team: Abidol and darunavir can inhibit coronavirus. Retrieved fromhttps://www.thepaper.cn/newsDetail_forward_5777936

REUTERS. (2020). Chinese doctors using plasma therapy on coronavirus, WHO says 'very valid' approach. Retrieved fromhttps://www.reuters.com/article/us-china-health-hospital/chinese-doctors-using-plasma-therapyon-coronavirus-who-says-very-valid-approach-idUSKBN20B1M6

rfi. (2020). Wuhan epidemic, official suggest No. 0 patient has nothing to do with fish market game market.

Riou, J., \& Althaus, C. L. (2020). Pattern of early human-to-human transmission of Wuhan 2019 novel coronavirus (2019-nCoV), December 2019 to January 2020. Euro Surveill, 25 (4). doi:10.2807/15607917.Es.2020.25.4.2000058

Rothe, C., Schunk, M., Sothmann, P., Bretzel, G., Froeschl, G., Wallrauch, C., . . Hoelscher, M. (2020). Transmission of 2019-nCoV Infection from an Asymptomatic Contact in Germany. N Engl J Med . doi:10.1056/NEJMc2001468

Savarino, A., Di Trani, L., Donatelli, I., Cauda, R., \& Cassone, A. (2006). New insights into the antiviral effects of chloroquine. The Lancet Infectious Diseases, 6 (2), 67-69. doi:10.1016/S1473-3099(06)70361-9

Sheahan, T. P., Sims, A. C., Graham, R. L., Menachery, V. D., Gralinski, L. E., Case, J. B., . . Baric, R. S. (2017). Broad-spectrum antiviral GS-5734 inhibits both epidemic and zoonotic coronaviruses. Sci Transl Med, 9 (396). doi:10.1126/scitranslmed.aal3653

Steenhuysen, J. K., Kate. (2020). With Wuhan virus genetic code in hand, scientists begin work on a vaccine. Retrieved fromhttps://www.reuters.com/article/us-china-health-vaccines-idUSKBN1ZN2J8

Su, S., Wong, G., Shi, W., Liu, J., Lai, A. C. K., Zhou, J., . . Gao, G. F. (2016). Epidemiology, Genetic Recombination, and Pathogenesis of Coronaviruses. Trends Microbiol, 24 (6), 490-502. doi:10.1016/j.tim.2016.03.003 
Vincent, M. J., Bergeron, E., Benjannet, S., Erickson, B. R., Rollin, P. E., Ksiazek, T. G., . . Nichol, S. T. (2005). Chloroquine is a potent inhibitor of SARS coronavirus infection and spread. Virology Journal, 2 (1), 69. doi:10.1186/1743-422X-2-69

Wang, F.-S., \& Zhang, C. (2020). What to do next to control the 2019-nCoV epidemic? The Lancet, 395 (10222), 391-393. doi:10.1016/S0140-6736(20)30300-7

Wang, M., Cao, R., Zhang, L., Yang, X., Liu, J., Xu, M., . . . Xiao, G. (2020). Remdesivir and chloroquine effectively inhibit the recently emerged novel coronavirus (2019-nCoV) in vitro. Cell Research . doi:10.1038/s41422-020-0282-0

Wang, Q., Wong, G., Lu, G., Yan, J., \& Gao, G. F. (2016). MERS-CoV spike protein: Targets for vaccines and therapeutics. Antiviral Res, 133 , 165-177. doi:10.1016/j.antiviral.2016.07.015

Warren, T. K., Jordan, R., Lo, M. K., Ray, A. S., Mackman, R. L., Soloveva, V., . . Bavari, S. (2016). Therapeutic efficacy of the small molecule GS-5734 against Ebola virus in rhesus monkeys. Nature, 531 (7594), 381-385. doi:10.1038/nature17180

WHO. (2020). Coronavirus disease 2019 (COVID-19) Situation Report - 82. Retrieved fromhttps://www.who.int/docs/defaultsource/coronaviruse/situation-reports/20200411-sitrep-82-covid-19.pdf? sfvrsn=74a5d15_2

Wired. (2020). The Coronavirus Is Now Infecting More People Outside China. Retrieved fromhttps://www.wired.com/story/th coronavirus-is-now-infecting-more-people-outside-china/

Wong, A. C. P., Li, X., Lau, S. K. P., \& Woo, P. C. Y. (2019). Global Epidemiology of Bat Coronaviruses. Viruses, 11 (2). doi:10.3390/v11020174

Wu, F., Zhao, S., Yu, B., Chen, Y.-M., Wang, W., Song, Z.-G., . . . Zhang, Y.-Z. (2020). A new coronavirus associated with human respiratory disease in China. Nature . doi:10.1038/s41586-020-2008-3

Xu, X., Chen, P., Wang, J., Feng, J., Zhou, H., Li, X., . . Hao, P. (2020). Evolution of the novel coronavirus from the ongoing Wuhan outbreak and modeling of its spike protein for risk of human transmission. Sci China Life Sci . doi:10.1007/s11427-020-1637-5

Xu, Z., Shi, L., Wang, Y., Zhang, J., Huang, L., Zhang, C., . . Wang, F.-S. Pathological findings of COVID-19 associated with acute respiratory distress syndrome. The Lancet Respiratory Medicine . doi:10.1016/S22132600(20)30076-X

Yan, Y., Zou, Z., Sun, Y., Li, X., Xu, K.-F., Wei, Y., . . Jiang, C. (2013). Anti-malaria drug chloroquine is highly effective in treating avian influenza A H5N1 virus infection in an animal model. Cell Research, 23 (2), 300-302. doi:10.1038/cr.2012.165

Yang, X. L., Hu, B., Wang, B., Wang, M. N., Zhang, Q., Zhang, W., . . . Shi, Z. L. (2015). Isolation and Characterization of a Novel Bat Coronavirus Closely Related to the Direct Progenitor of Severe Acute Respiratory Syndrome Coronavirus. J Virol, 90 (6), 3253-3256. doi:10.1128/jvi.02582-15

Yang, Y., Lu, Q., Liu, M., Wang, Y., Zhang, A., Jalali, N., . . . Fang, L. (2020). Epidemiological and clinical features of the 2019 novel coronavirus outbreak in China. medRxiv , 2020.2002.2010.20021675. doi: $10.1101 / 2020.02 .10 .20021675$

YSA, Y. S. U. (2020). New progress in new crown pneumonia.

Zhe, W. Q. R. (2020). Disinfection measures for new coronavirus-infected pneumonia epidemics in 2019. Chinese Journal of Disinfection (01), 1-4.

Zhou, P., Yang, X.-L., Wang, X.-G., Hu, B., Zhang, L., Zhang, W., . . . Shi, Z.-L. (2020a). A pneumonia outbreak associated with a new coronavirus of probable bat origin. Nature . doi:10.1038/s41586-020-2012-7 
Zhou, P., Yang, X.-L., Wang, X.-G., Hu, B., Zhang, L., Zhang, W., . . . Shi, Z.-L. (2020b). A pneumonia outbreak associated with a new coronavirus of probable bat origin. Nature, 579 (7798), 270-273. doi:10.1038/s41586-020-2012-7

Zhou, Y., Yang, Y., Huang, J., Jiang, S., \& Du, L. (2019). Advances in MERS-CoV Vaccines and Therapeutics Based on the Receptor-Binding Domain. Viruses, 11 (1). doi:10.3390/v11010060

Zhu, N., Zhang, D., Wang, W., Li, X., Yang, B., Song, J., . . Research, T. (2020). A Novel Coronavirus from Patients with Pneumonia in China, 2019. The New England journal of medicine, 10.1056/NEJMoa2001017. doi:10.1056/NEJMoa2001017

Table 1 Summary of some drugs reported being effective in treating SARS-CoV-2 $\S$

\begin{tabular}{lll}
\hline & Name & Original use \\
\hline Drugs reported effective & Remdesivir & Broad-spectrum RNA + virus antiviral drug (SARS/MERS/Ebo \\
& Chloroquine & Treats Malarial and autoimmune disease \\
& Hydroxychloroquine & Treats Systemic lupus erythematosus \\
& Favipiravir & Anti-Influenza \\
& Arbidol & Anti-Influenza \\
& Darunavir & Anti-HIV ++ \\
& Chinese Traditional Medicine &.$\cdot$ \\
\hline
\end{tabular}

All abbreviations: SARS-CoV- $2^{\S}=$ Severe acute respiratory syndrome coronavirus- $2 ; \mathrm{RNA}^{+}=$Ribonucleic Acid; $\mathrm{HIV}^{++}=$Human Immunodeficiency Viruses; $\mathrm{PH}^{\mathrm{P}}=$ potential hydrogen.

\begin{tabular}{lll}
\hline & Original use & Name \\
\hline Candidate drugs & Anti-HIV ${ }^{+}$ & Indinavir, Saquinavir, Lopinavir, Carfilzomib, Ritonavir, \\
& Chinese herbal extract & Shikonin, deoxidant emodin, polydatin, Shandougen Chal \\
& Anticancer & Bortezomib, Carmofur, PX-12 \\
Anti-respiratory syncytial virus & Enzaplatovir, Presatovir \\
Treats neurodegenerative diseases & Tideglusib, TDZD-8 \\
Anti-virus that targets human macrophage & Maribavir \\
Anti-SARS-CoV & Cinnamylamine \\
Immunosuppressant & Cyclosporin \\
Anti-inflammatory & Ebselen \\
Anti-asthma & Montelukast \\
Treats alcohol abuse & Disulfiram \\
Anti-HBV/HCV ++ & PEG-interferon $\alpha-2 \mathrm{a}$ and $\alpha-2 \mathrm{~b}$ \\
Broad-spectrum RNA $\mathbb{I}$ virus antiviral drug & Ribavirin, Galidesivir \\
\hline
\end{tabular}

\section{Table 2 Summary of some candidate drugs of SARS-CoV-2 $\S$}

All abbreviations: SARS-CoV-2 ${ }^{\S}=$ Severe acute respiratory syndrome coronavirus- 2 ; $\mathrm{HIV}^{+}=$Human Immunodeficiency Viruses; $\mathrm{HBV}^{/} \mathrm{HCV}^{++}=$Hepatitis $\mathrm{B}$ virus $/$ Hepatitis $\mathrm{C}$ virus; $\mathrm{RNA}^{\mathrm{P}}=$ Ribonucleic Acid; 